\title{
RESEARCH DIRECTIONS REGARDING THE ADOPTION OF FORMAL METHODS IN THE RAILWAY SIGNALING SECTOR: DETERMINANTS AND NEXT STEPS FOR FUTURE-PROOF RAILWAYS
}

\author{
DIMITRIOS RIZOPOULOS ${ }^{1}$, NILS O. E. OLSSON ${ }^{2}$, ANDERS LINDAHL $^{1} \&$ OLOV LINDFELDT $^{3}$ \\ ${ }^{1}$ KTH Royal Institute of Technology, Sweden \\ ${ }^{2}$ NTNU Norwegian University of Science and Technology, Norway \\ ${ }^{3}$ MTR Pendeltågen, Sweden
}

\begin{abstract}
The terms Formal Methods (FMs) refer to a set of techniques and software toolkits that, based on mathematical rigor, can enhance safety, security, and the efficient operation of a wide range of systems. Considering that several innovative FMs applications have been performed over the last few decades, the utility of toolkits that are based on FMs has already been showcased in several industrial settings, such as the avionics and automotive industries, medical devices, computer software, and hardware systems, and finally, the railways and the railway-signalling sector. The current article focuses on the last of the aforementioned sectors, that of railway signalling, and aims to analyse research directions that regard the adoption of FMs in signalling. Despite the benefits and the availability of reports on the topic, the implementation of the adoption of FMs can be considered yet to be successful in most organizations that develop related systems. The authors have observed that this implementation lag in the adoption of FMs may stem from the absence of systematic approaches to the study of adoption. In that regard, a set of five Research Questions (RQs) is introduced in this article for the adoption of FMs in railway signalling systems. Furthermore, answers regarding two of those questions are given within the current article regarding: (i) studies on the adoption of FMs, and (ii) successful applications and benefits of FMs. The remaining three RQs are intended to point out issues for future research. By providing answers to all five RQs, it is expected that a map for the demand of FMs applications in railway signalling is introduced in the following years, as well as the role of the potential stakeholders in those applications.
\end{abstract}

Keywords: formal methods, railway signalling, technology adoption.

\section{INTRODUCTION}

Various meanings have been considered for the term innovation. In the study by Edison et al. [1], several definitions have been analyzed for coverage against aspects of the meaning of innovation, as identified in the scientific literature. The results showed that the most suitable was given in the article by Crossan and Apaydin in 2009 [2], who state that: "Innovation is: production or adoption, assimilation, and exploitation of a value-added novelty in economic and social spheres; renewal and enlargement of products, services, and markets; development of new methods of production; and establishment of new management systems. It is both a process and an outcome". With adoption being one of the key aspects of innovation, once an innovative method is identified, its implementation within organizations can take place, with its diffusion taking place from an individual or a group who may be called innovators, to other groups that can adopt the innovation and can possibly benefit from its utilization. While the aforementioned diffusion and adoption process of different innovations can take place across many organizations in several stages [3], the attempt to incorporate innovations to the way the companies operate can be proven to be a trivial task.

The current article discusses the adoption of FMs or semi-Formal Methods (semi-FMs) tools and toolkits, as an innovative technology, to the sector of railway signaling. Regarding 
FMs, the terms include all the mathematical based methods that allow the rigorous specification of cyber-physical systems, their formal development, and verification through mathematical proof. In more detail, and concerning the railway-signaling sector and the traffic control systems, some of the benefits that FMs enable are:

- The enhancement of safety and security in railways thought the formal specification, modeling, development, and verification of signaling systems, in fully operational or degraded operation scenarios;

- Automated development of verified signaling systems and sub-systems that comply with international standards;

- Improvement of the specification, design, and architecture of a system, primarily through FMs or semi-FMs application in the early stages of a project life cycle;

- Re-usability of artifacts from a single product development process into multiple projects;

- Rigorous specification of requirements in procurement tasks;

- The standardization of protocols, the reduction or improvement of interfaces between signaling systems and sub-systems, and the efficient management of increasing complexity and automation;

- The cost reduction and schedule compression of projects' through the application of FMs or semi-FMs on multiple levels of the project.

Secondary long-term improvements in the signaling systems themselves can also affect the train services, with services' punctuality [4] or track capacity [5] being directly connected to signaling systems in previous studies. Despite the potential benefits, the adoption of FMs in railway signaling can be considered not to be complete yet as organizations hesitate to invest or utilize FMs tools in their projects. On a higher level, this lag in the adoption can be partially attributed to the general misconceptions that regard FMs regardless of the application sector. They are related to the perceived ease and benefits of use or even concerns regarding the support that FMs tools usually get from their developers. Those generic, as we may call them, misconceptions that experienced professionals in the field of FMs are already aware of, have been available in the scientific literature since 1990 in reviews [6], [7].

In order to extract the benefits of the utilization of FMs, product development routines, as followed by railway signaling companies, would need to include FMs tools or toolkits at some of their stages. The FMs toolkits, do not necessarily have to be the scientific methods themselves but can have a lower or higher level of abstraction. Although there are several mature studies [8]-[11] that review FMs tools and applications, there are only a few reports [12]-[17] that address the adoption of FMs, or include references to adoption and implementation.

The purpose of this article is to identify appropriate Research Questions (RQs) for further research regarding FMs adoption in railway signaling. Based on observations from both the academic and industrial settings, a set of five questions is given throughout this article, with two being answered within this article. Those first two RQs are:

1. What is the current state of adoption of FMs in railway signaling?

2. What are the potential benefits of FMs applications in railway signaling?

The latter three questions, and proposing ways to address them, are included in the conclusion. All of the five (first two and latter three) questions that are proposed in this article are defined on a general level so that future research is not constrained by the scope of those directions, as they are presented in this paper, but is instead guided within the goals that need 
to be attained. Our proposal is that those questions and answers are provided as a Layman's report in the field of FMs application to railway signaling.

Our method for the derivation of insights and results presented within this article is a literature review that sourced articles, reports, and books from several platforms such as ScienceDirect, Google Scholar, ResearchGate, and Web of Science. In that regard, Section 2 of this paper discusses the current state of adoption of FMs in railway signaling systems development. Section 3 gives an overview of applications that exist within signaling, where potential benefits are showcased from mainly the academic but also the industrial environment. Finally, in the last concluding section, we provide an overview of what has been discussed and what are some further research directions.

\section{THE CURRENT STATE OF ADOPTION OF FORMAL METHODS IN SIGNALING}

Regarding the adoption of FMs in railway signaling, it is a phenomenon that has not yet been studied in great depth from the academic perspective, and only very few articles can be found that address or even discuss this problem. All of the works tend to share empirical knowledge that has been gained through projects. Thus, the authors have observed that a systematic study of adoption is a research gap in the literature.

Let us first present the existing work. The first article by Bacherini et al. [12], several insights are presented regarding adoption that stem from the attempt to disseminate FMs tools in the railway signaling division of General Electric Transportation Systems (GETS) in collaboration with academic experts, when the problem of certifying software components within the CENELEC guidelines appeared. While several tools were considered, the main aim was to establish and disseminate knowledge about the semi-formal Stateflow tool of Matlab within GETS. As mentioned in the paper, the compatibility of Stateflow with other Matlab and Simulink tools, and the already existing experience in Matlab usage within the company, has been proven to be beneficial factors. At the time of the publication of the article, the authors discuss that while the adoption is not full yet, it is implemented on a project by project base. They point out that several factors benefited the process of adoption, such as: (i) collaboration with academia; (ii) prolonged time-to-market phase, and thus, more time for experimentation; (iii) the pressure as perceived by the organization and professionals to comply with CENELEC; (iv) the general market evolution; and (v) the pressure, as exercised by clients to include rigorous and formal specifications.

Another article was published in 2013 by Ferrari et al. [16], who share the challenges and learning lessons that GETS faced in adopting model-based development regarding the railway signaling systems developed at the company, in contrast to code-based processes that have been traditionally followed. One of the concluding remarks about the adoption of FMs and semi-FMs in GETS is that it should happen in steps, with the early stages focusing on less formal tasks and later move on to use FMs for more difficult tasks. Interestingly, here the authors point out the importance of a multi-stage approach regarding the technical aspect of adoption, so that industrial practitioners can assimilate the new knowledge on a task by task basis. We would like to state that this opinion resembles our thoughts, and further augments the already well-received multi-stage paradigm of analyzing technology adoption [3].

In the same year, Ferrari et al. [18] have published an article on a case study on the development of the driving support Automatic Train Protection (ATP) system Metrô Rio according to a formal model-based process, as well as another report [17] to discuss adoption regarding the project described in [18] alongside another two projects dealing with FMs application to ATP systems. 
Additionally, in work by Huhn and Milius [13], insights are given regarding adoption, and the impression of the industrial partner to the case study presented for a Level Crossing (LC, LCs) controller system. The authors highlight that human expertise and perceived effort are two of the most critical barriers to adoption.

Moving on to the second article by Basile et al. [14], where the authors present the results of questionnaires that have been conducted within the frame of ASTRail project that concern the industrial uptake of FMs in railways. More specifically, the criteria for this questionnaire were (i) understanding adoption (i.e., uptake) of FMs and (ii) discover the features that the railways professional considered as more vital (i.e., relevant) for the application of FMs. The questionnaire was addressed to the participants of RSSRail17, and the results show that the B method is one of the most well-established tools and that the maturity of the tools, the learnability, and the documentation were among the most essential features. In this survey, the cost of the tools was not considered to be highly connected to the adoption of FMs since only $13.6 \%$ of the respondents consider "low-cost" as an attributable determinant for the use of the corresponding FMs toolkits.

Next, we will refer to the work by ter Beek et al. [15], where a summary of the results of three questionnaires that regard adoption are given that were conducted within ASTRail and X2Rail-2. Two of the questionnaires were conducted within X2Rail-2 and another one in ASTRail project (same with Basile et al. [14]). As given in the conclusion of the study [15], the results were harmonized and can be summarized in the following key points: (i) Most FMs applications are focused on interlocking systems within organizations; (ii) FMs are mainly used for formal specification and verification; (iii) The B-family tools are the most well-established with SCADE and Simulink being also already well-received; (iv) Essential features of tools are considered formal verification, formal modeling, traceability, simulation, test-generation and, finally, code generation; (v) regarding the adoption of tools, the most important determinants were documented to be tool maturity, usability, and learnability of the tools. An interesting insight also provided in the same article is that the industrial perception of FMs tends to fluctuate and is not solid, with FMs may sometimes be confused with semi-FMs. This insight confirms in a way our previous statement in this article that, possibly, similar misconceptions [6], [7] that exist for FMs on a general level, also, exist within railway signaling.

Overall, in this section all reports and articles have been included which discuss the adoption of FMs in railway signaling, and that can be found on online bibliographic databases at the time of publication of this article. One can notice a cohesion in the results of the articles in the literature of FMs adoption in railway signaling. From those observations, it could be claimed that it is only now that professionals in the field are starting to accumulate and formalize the knowledge that has been acquired through research in both the academic and the industrial settings regarding how FMs toolkits can be disseminated into organizations.

\section{STUDIES AND APPLICATIONS THAT UTILIZE FORMAL METHODS IN RAILWAY SIGNALING}

One of the driving forces for utilizing FMs in railway signaling the fail-safe operation principle. In other terms, signaling equipment must be guaranteed to operate the trains safely, even when some of the sub-systems have failed, so that human lives are not at stake. This is one of the main reasons for which signaling systems are categorized as safety-critical systems and are subject to several safety guidelines and imposed standards for their development. The most prominent related example is EN50128 guidelines by the European Committee for Electrotechnical Standardization (CENELEC). Although some professionals claim that the 
demand for FMs applications stems only from legislation, it is now obvious more than ever that there are other use-cases outside legislative requirements, for which FMs can be proven beneficial. In the next few Subsections 3.1 to 3.8, a review of articles and reports on studies and applications that have showcased beneficial results for the systems is given. The value-added result of the following review is not to be exhaustive in terms of articles within each application field, but its goal is instead to assemble all applications and use cases, for which articles and reports can be found within the scientific literature.

\subsection{Level crossings (LC, LCs)}

Although the terms railway level crossing could refer to the physical location where the railway line crosses the road or pedestrian transport network, in this subsection of the article we will refer to studies that report on successful applications of FMs approach to the safety-critical cyber-physical systems and sub-systems of the LCs that ensure the proper function of the equipment. Regarding LCs, one of the main aims of applying FMs is the assessment of safety, especially in the case of failed components locally or across the network. Of great importance is to reassure that the LC can work as an independent system and guarantees fail-safe operation as an automatic system since it is considered to be a black spot for railway transportation systems.

An approach to LC verification of safety that is worth mentioning is the one by Mekki et al. [19] that came in 2012. In their article, they focus on two hazardous situations that are connected to several LC accidents, and though the specification by formal notation and the verification by model-checking, they validate a new architecture for LC that can deal with those two specific situations. Another set of studies on LC is included by Mohamed Ghazel [20], [21]. A Petri net model-based simulation approach to the risk assessment of various dynamics (i.e., collision cases) within the LC environment is introduced in 2009 in the first article [20], where the model occurs progressively by integrating other simpler parts of the LC and their behavior, whether it regards how road or rail traffic affects the overall LC. Finally, we refer to the last study by Ghazel [21], where the author proposes a new LC control/command architecture in the context of ERTMS L2/3 operation. The article provides some beneficial insights regarding FMs capabilities, which on the one hand, can be the elimination of on-site testing, given that enough practical constraints are accommodated, or, on the other hand, the validation of new architectures that can be more efficient (fewer sensing devices).

\subsection{Systems related to moving block}

The moving block mode of operation for a railway network or line is the case when the signaling systems enable the fragmentation of routes of the network into moving blocks, in real-time, with the support of computer systems that consider parameters such as train positions, speeds, acceleration, and braking distances. While the operation of moving block lines is already applied at several transportation systems (London Underground and New York City Subway), it can be considered that further specification work could be useful for its global acceptance and application to networks.

FMs have been used for the performability evaluation of the ERTMS/ECTS Level 3 system in work by Biagi et al. [22]. The authors introduce an approach to evaluate emergency stops of a system under ERTMS/ECTC Level 3 operation that are caused by a combination of communication failures that could be burst noise in communication links and connection losses due to failure of devices or cell handovers when reaching borders of GSM-R cells. In 
the work by Hansen et al. [23], a full executable application based on Hybrid ERTMS/ECTS Level 3 (HBL3) is presented. HBL3 operational scheme does fully utilizing moving block functionality but instead divides the sections into virtual subsections which are used as fixed blocks, but provide finer granularity, as compared to total fixed block operation (such as in the case of ERTMS/ECTS Level 2). Insights are provided about FMs tools support as well as for the HBL3 as a system, too. A Virtual Block Function (VBF) is included in the approach, which behaves as an interlocking that transmits virtual signals to the RBC. In contrast, the implementation of the whole approach, by B Method family tools, can be considered as an executable reference specification for the demonstration and understanding of HBL3 and its core principles.

In the article by Qian et al. [24], a SCADE based development process is showcased to model and verify the Zone Controller of CBTC that enables the moving block function by calculating movement authority of trains. The authors of this article suggest a way to deal with the state explosion problem that occurs when many real systems are modeled and then verified according to FMs approaches. In general, the state explosion problem is considered to be a bottleneck for FMs applications to real systems; thus, solutions regarding it are beneficial within railway signaling applications. In more detail, they propose slicing criteria that can simplify the complexity of the SCADE models and can verify the correctness of movement authority calculation for a given train network. Such slicing criteria, and similar decomposition approaches, are deemed to be necessary for the efficient execution of calculations that are based on complicated models of vast size in other adjacent computer science applications fields [25]. Similarly, studies like the one by Qian et al. [24] could be necessary to be expanded in order to enable the construction and verification of models of signaling systems of large sizes.

\subsection{Automatic Train Protection/Control (ATP/ATC) functions/systems}

Those systems are generally considered to be protection systems that include speed and position control mechanisms that function based on inputs from external sources such as track-side equipment. They are used to guarantee safe speeds, braking distances, and breaking system triggering of the vehicles concerning the braking curve of the trains. They can be implemented as driver support systems, or they can be systems that coordinate the driverless operation of trains. ATP/ATC can be used to refer to the overall function of those systems, including both track-side (i.e., wayside) and on-board equipment. FMs can be used here to assure the safe operation of trains in fully operational scenarios or degraded scenario when some of the components of the system do not work as expected.

Successful applications can be found since 1993 [26], but other modern approaches can be found in the literature, such as the work by $\mathrm{Wu}$ et al. [27], where a mathematical problem is built-in ANSYS's SCADE Suite to calculate the dynamic curves of the ATP system. The curves are modeled and verified through several techniques such as a simulation test, a model test coverage (according to test cases), and formal verification (through SCADE's integrated tool). Another approach to FMs application on ATP systems has been presented by Ferrari et al. [18], wherein their case study Simulink and Stateflow have been used to produce a formal model of on-board ATP equipment of the Metro Rio Automatic Train Protection system. The wayside objects are modeled as an encoder and a transponder that provide the processed data to the on-board system, which is of the main interest in this study. Among the benefits that are reported in this study is the reduction of the bugs found during development by $71 \%$ and the average time of bug detection and correction from almost an hour to less than 30 minutes per bug. 


\subsection{Automatic Train Operation (ATO) function}

Another notable instance of a successful application is the application of FMs to the ATO system for the Paris Metro line 14 (under the Meteor project/automatic train control system). A report by Behm et al. [28] presents processes followed for the development of the safety-critical software of this line, as well as insights on the organization and roles of teams within the project. Behm et al. [28] state in their conclusions that the application of FMs in the Meteor project has been considered cost-effective, with the increase in quality being noticeable even before systems' maintenance costs are considered. An even further successful application of the B method is reported in the paper by Essamé et al. [29] for the New York City Canarsie Line, whose critical safety software development was proven to be more demanding than that of the Meteor system. The study by Essamé et al. [29], which can be considered a continuation study when compared to the one by Behm et al. [28], states that $\mathrm{B}$ method tools are incorporated in the development of safety-critical software within the organization.

\subsection{Communications-Based Train Control (CBTC)}

Under the terms of CBTC, all signaling systems are included that are used for the communications between the trains running on a track of the network and the track-side equipment. CBTC systems are comprised of several software and hardware sub-systems that are adapted to each application, with both on-board and track-side equipment. In some cases, this adaptation happens through configuration data, which are considered to describe the arrangements of the equipment and their functions alongside the rail infrastructure. In contrast to dynamic data, static configuration data (e.g., geographical arrangement of the equipment) never change, and their safe operation and compliance with safety and functional requirements must be guaranteed in the initial life cycles of a project. Successful applications of FMs on configuration data have been conducted in the past [30]. FMs applications on CBTC systems can be found for other mechanisms/parts of the CBTC system, such as track circuits or emergency cancellation functions, as reported in the article by Comptier et al. [31]. In this article, it is described how Event-B and Atelier B were utilized to perform rigorous safety analysis of the multi-supplier interoperable Octys (Open Control of Train Interchangeable and Integrated system) CBTC system which has been deployed on Paris metro lines 3, 5 and 9 starting 2010 as reported in the article.

\subsection{Railway interlocking systems}

A railway interlocking system, or as it usually called interlocking(s), is one of the systems responsible for the safe guidance of trains through the network and is assigned with Safety Integrity Level 4 (SIL4) within the CENELEC EN50128 guidelines. As stated above, SSIL 4 is the highest level of safety integrity, as described in EN50128. Interlockings allow the movement of trains according to a set of rules that affect track-side elements. These movement authorities that are provided to train have to occur according to a specific way, or as it widely called according to interlocking logic. Interlocking logic has to be mathematically examined and proved to reassure the fail-safe operation of the network. Several successful applications have been showcased for interlocking, which can be considered a leading example in the field of FMs applications to railway signaling. It has been decided to not explain interlocking articles and reports in further detail, as instances of successful 
application can be easily found both within the academic environment [8], [32], but also from an industrial setting [33].

\subsection{A short reference to other systems in railways}

Some other instances of successful application can be found for the railways industry. One example is the work reported by Van Tang et al. [34], who report the application of FMs framework called the Vienna Development Method (VDM++) on a Train Fare Calculation and Adjustment System. These kinds of systems are the ones utilized to assist passengers at acquiring tickets at the right price for their trips within the transportation network. Because those systems tend to serve vast numbers of passengers on a daily basis, a small error in the system can lead to significant losses in short amounts of time for either the passengers or the operators. As in the case of other signaling systems mentioned above, the existence of errors once a system is deployed can lead to the need to fix those errors while the system is on-line and running, which can be a hard task for all stakeholders involved, such as software companies, services operators, infrastructure managers, and governments. In the case study, as presented [34], for a Train Fare Calculation and Adjustment System in Japan, the application of VDM++ has been viewed as very successful as it allowed the developers to come up with 32 erroneous issues in the informal specification document for the system that uses both newly developed modules but also legacy ones from an existing fare calculation system.

Platform Screen Doors (PSD) is another system that FMs have been applied to. PSD are the doors that are used to prevent passengers or users of rail or metro services from falling on to the track. Their use is of great importance for automatic/autonomous trains that do not have an on-board crew that manually checks whether all passengers are aboard. Also, their performance, correct operation, and relation to dwell times of services at stations, even in non-erroneous situations, has been linked to a decrease in capacity in some cases of study [35]. Thus, such a system is uniquely in need of certified proof of proper function to facilitate modern automatic/autonomous railway systems. A successful application of FMs on such a system has been implemented in the past [36], where the authors discuss an application where functional and safety requirements of the PSD and its controller software were verified. In the same article, it is reported that, at the time of publishing, the system successfully operated for eight months, controlling 96000 trains, without a single fault in its operation.

\subsection{Security of railway signaling systems}

Most of the articles and reports referenced in Section 3 of this article refer to the safety aspect of signaling systems and reassuring that the fail-safe operation of a railway line is never compromised, even in cases when some of the components fail. However, since railway signaling systems are mixed-criticality systems, their web resilience has to be evaluated and guaranteed in all cases. With the increase of phenomena like cyber-terrorism and malevolent attacks in general, FMs can be used to create fully secure architectures that allow the proper function of the railway network, while in the same time they enable the developers to make sure that security requirements are not violated across the engineering and development processes. Reports can be found on such cases, such as the one by Heinrich et al. [37], who propose several security requirements that architectures, such as the one proposed in this article, should cover.

Overall, the scope of Section 3 was to provide a comprehensive report, or a map, as we would like to call it, of FMs studies and applications in railway signaling (a few adjacent 
fields within railways). The goal for that section was to provide an answer to RQ 2 as provided in the introduction and provide a Layman report for what is possible within railway signaling by using FMs.

\section{CONCLUDING REMARKS \& FURTHER RESEARCH QUESTIONS}

The current situation regarding FMs and their adoption to railway signaling applications can be considered to be in a stage where FMs are getting more widely acknowledged, distributed, and used over the years, but are not deeply integrated into systems development life-cycles yet at this point, and not utilized to their full potential. While there are enough tools and some successful applications, the understanding of the diffusion of their adoption remains a trivial task. Besides, from previous studies, it is noticeable that the use of a standard toolkit has not arisen yet, and different organizations use different approaches according to each team's or engineer's perspective and background. To gain a better understanding of FMs adoption, and from the literature study and several observations as presented in this article, the following five RQs arise:

1. What is the current state of adoption of FMs in railway signaling?

2. What are the potential benefits of FMs applications in railway signaling?

3. What is the current and future demand for FMs applications in railway signaling, and who are the stakeholders in the existing and potential projects?

4. What are the determinant factors of user acceptance of FMs as a new technology in organizations in railway signaling?

5. What are the benefits for organizations that invest in FMs tools and their adoption regarding cost reduction and schedule compression of signaling projects?

The first RQ question is answered by Section 2 of this article. From all of the available material that discusses adoption, it can be noticed that some potential crucial factors for adoption are: (i) adoption should be studied from both an academic and industrial setting; (ii) definition of the learning curve (considering ease of use, documentation and available support) for toolkits can be beneficial, especially on an individual user perspective; (iii) compatibility across tools of toolkits can be substantial; (iv) on a general level, formal specification, formal verification, and formal modeling are the most relevant features, as observed from questionnaire-based studies.

The second RQ is answered in this article, too, in Section 3. A comprehensive review is given, where articles and reports on studies and applications that showcase potential benefits have been presented. Overall, the main takeaway regarding this second question is that successful applications exist within the academic sector, but what would be more interesting is to see how those benefits that stem from academia translate into real-world practice and industrial applications. Reports on industrial projects can be beneficial for the dissemination of knowledge within railway signaling.

Regarding RQ three, it is a research question that is expected to be answered by an expert panel on FMs applications to signaling. The value-added result in the potential answer has to be supported by data on current and future projects. Discussion of stakeholders and their role in projects and applications could be highlighted.

Regarding RQ four, we propose the study of determinants of user acceptance of FMs within railway signaling. User acceptance by individual professionals within the sector is an important step, as also mentioned by Kamal [3]. In order to conduct such a systematic study of an adoption of a technology, several theoretical lenses can be used, such as the work by Venkatesh et al. [38] on the Unified Theory of Acceptance and Use of Technology (UTAUT). In UTAUT, the authors capture the most practical and essential aspects of eight previous 
models and consider four key constructs, and the relationship between them, as the most critical determinants for user intentions to utilize a new information system and their corresponding usage behavior. Namely, the constructs are (i) performance expectancy, (ii) effort expectancy, (iii) social influence, and (iv) facilitating conditions, while the research model also accounts for the role of key moderators such as gender, age, voluntariness, and experience. Regarding the application of such a framework, in the case of UTAUT, one could claim that is the most prominent framework used in modern literature with the original paper being cited 27529 times, several examples can be taken from previous studies that study technologies that range from course management software to internet banking. Given its widespread acceptance and cross-field compatibility, UTAUT can be proven to be suitable to the case of FMs application to railway signaling. By systematically studying the determinants of user acceptance in the case of signaling systems in railways, it is expected that a solid understanding of the structure of adoption will be gained in this direction of research. While the railways' sector is considered to be a conservative one, getting insights on which determinants will allow individuals to be more open to using new technologies can be beneficial not only in the case of FMs but in other cases of technology adoption in railways, such as machine learning and blockchain technologies.

Finally, there is RQ five, which concerns the economic aspect of FMs adoption and is a longer-term study to be conducted. It is proposed that this topic will be studied from a simplified perspective of individual signaling projects and how did they benefit in two aspects, the first being (i) cost reduction and the second being (ii) schedule compression. Although other aspects of projects could be measured too, we assume those two to be the main interests of stakeholders.

Moving forward and regarding the future, we consider that FMs will be applied in more and more projects over the years. Concerning prospective upgrade of the European railways' network to comply with higher standards of CBTC and ERTMS, and the interoperability of trains in the European Union, FMs is one of the essential scientific disciplines, if not the most important one, in the attempt to achieve this compliance with international standards and compliance in between networks of different countries. Applications will also vary to the level of abstraction of their application regarding the knowledge that is required to apply the tools. Some high-level tools will be necessary for most of the projects, but also low-level tools will be essential to guarantee the formal development and formal verification of properties of specific sub-systems.

\section{REFERENCES}

[1] Edison, H., bin Ali, N. \& Torkar, R., Towards innovation measurement in the software industry. Journal of Systems and Software, 86(5), pp. 1390-1407, 2013.

[2] Crossan, M.M. \& Apaydin, M., A multi-dimensional framework of organizational innovation: A systematic review of the literature. Journal of Management Studies, 47(6), pp. 1154-1191, 2010 Sep.

[3] Kamal, M.M., IT innovation adoption in the government sector: Identifying the critical success factors. Journal of Enterprise Information Management, 19(2), pp. 192-222, 2006.

[4] Palmqvist, C.W., Olsson, N.O.E. \& Hiselius, L.W., Some influencing factors for passenger train punctuality in Sweden. International Journal of Prognostics and Health Management, 8(7), 2017.

[5] Goverde, R.M.P., Corman, F. \& D'Ariano, A., Railway line capacity consumption of different railway signalling systems under scheduled and disturbed conditions. Journal of Rail Transport Planning \& Management, 3(3), pp. 78-94, 2013. 
[6] Hall, A., Seven myths of formal methods. IEEE Software, 7(5), pp. 11-19, 1990.

[7] Bowen, J.P. \& Hinchey, M.G., Seven more myths of formal methods. IEEE Software, 12(4), 1995.

[8] Haxthausen, A.E., An introduction to formal methods for the development of safety-critical applications, DTU Informatics Technical University of Denmark, 2010.

[9] Gruner, S., Kumar, A. \& Maibaum, T., Towards a body of knowledge in formal methods for the railway domain: Identification of settled knowledge. Communications in Computer and Information Science, Springer, 2016.

[10] Fantechi, A., Flammini, F. \& Gnesi, S., Formal methods for railway control systems. International Journal on Software Tools for Technology Transfer, 16, pp. 643-646, 2014.

[11] ter Beek, M.H., Fantechi, A., Ferrari, A., Gnesi, S. \& Scopigno, R., Formal Methods for the Railway Sector, ERCIM NEWS 112., 2018.

[12] Bacherini, S., Fantechi, A., Tempestini, M. \& Zingoni, N., A story about formal methods adoption by a railway signaling manufacturer. Lecture Notes in Computer Science (including subseries Lecture Notes in Artificial Intelligence and Lecture Notes in Bioinformatics), pp. 179-189, 2006.

[13] Huhn, M. \& Milius, S., Observations on formal safety analysis in practice. Science of Computer Programming, 80, pp. 150-168, 2014.

[14] Basile, D. et al., On the industrial uptake of formal methods in the railway domain. Lecture Notes in Computer Science (including subseries Lecture Notes in Artificial Intelligence and Lecture Notes in Bioinformatics), pp. 20-29, 2018.

[15] ter Beek et al., Adopting formal methods in an industrial setting: The railways case. International Synopsium on Formal Methods, pp. 762-772, 2019.

[16] Ferrari., A., Fantechi, A., Gnesi, S. \& Magnani, G., Model-based development and formal methods in the railway industry. IEEE Software, 30(3), pp. 28-34, 2013.

[17] Ferrari, A., Fantechi, A. \& Gnesi, S., Lessons learnt from the adoption of formal model-based development. Lecture Notes in Computer Science (including subseries Lecture Notes in Artificial Intelligence and Lecture Notes in Bioinformatics), pp. 24-38, 2012.

[18] Ferrari, A., Fantechi, A., Magnani, G., Grasso, D. \& Tempestini, M., The Metrô Rio case study. Science of Computer Programming, 78(7), pp. 828-842, 2013.

[19] Mekki, A., Ghazel, M. \& Toguyeni, A., Validation of a new functional design of automatic protection systems at level crossings with model-checking techniques. IEEE Transactions on Intelligent Transportation Systems, 13(2), pp. 714-723, 2012.

[20] Ghazel, M., Using stochastic petri nets for level-crossing collision risk assessment. IEEE Transactions on Intelligent Transportation Systems, 10(4), pp. 668-677, 2009.

[21] Ghazel, M., A control scheme for automatic level crossings under the ERTMS/ETCS Level 2/3 Operation. IEEE Transactions on Intelligent Transportation Systems, 18(10), pp. 2667-2680, 2017.

[22] Biagi, M., Carnevali, L., Paolieri, M. \& Vicario, E., Performability evaluation of the ERTMS/ETCS - level 3. Transportation Research Part C: Emerging Technologies, 82, pp. 314-336, 2017.

[23] Hansen, D. et al., Validation and real-life demonstration of ETCS hybrid level 3 principles using a formal B model. International Journal on Software Tools for Technology Transfer, 22, pp. 315-332, 2020. 
[24] Qian, J., Liu, J., Chen, X. \& Sun, J., Modeling and verification of zone controller: The SCADE experience in China's railway systems. 2015 IEEE/ACM 1st International Workshop on Complex Faults and Failures in Large Software Systems (COUFLESS), pp. 48-54, 2015.

[25] Saharidis, G.K.D. \& Fragkogios, A., Open problems on benders decomposition algorithm, pp. 305-317, 2018.

[26] DaSilva, C., Dehbonei, B. \& Mejia, F., Formal specification in the development of industrial applications: Subway speed control system. Proceedings 5th IFIP Conference on Formal Description Techniques for Distributed Systems and Communication Protocols (FORTE 1992), Perros-Guirec, pp. 199-213, 1993.

[27] Wu, Q., Pan, T., Xue, X., Zhang, F. \& Jia, J., Modeling and implementation for dynamic curves of onboard automatic train protection system based on SCADE. Lecture Notes in Electrical Engineering, pp. 93-103, 2016.

[28] Behm, P., Benoit, P., Faivre, A. \& Meynadier, J.M., Météor: A successful application of b in a large project. Lecture Notes in Computer Science, pp. 369-387, 1999.

[29] Essamé, D. \& Dollé, D., B in large-scale projects: The canarsie line CBTC experience. Lecture Notes in Computer Science, pp. 252-254, 2006.

[30] Abo, R. \& Voisin, L., Formal implementation of data validation for railway safetyrelated systems with OVADO. Lecture Notes in Computer Science, pp. 221-36, 2014.

[31] Comptier, M., Deharbe, D., Perez, J.M., Mussat, L., Pierre, T. \& Sabatier, D., Safety analysis of a CBTC system: A rigorous approach with Event-B. Lecture Notes in Computer Science, pp. 148-159, 2017.

[32] $\mathrm{Vu}, \mathrm{L} . \mathrm{H}$., Formal development and verification of railway control systems: In the context of ERTMS/ETCS Level 2, Technical University of Denmark, 2015.

[33] Borälv, A., Interlocking design automation using prover trident. Lecture Notes in Computer Science, pp. 653-656, 2018.

[34] Van Tang, N., Souma, D., Hatayama, G. \& Ohsaki, H., Modeling and validating the train fare calculation and adjustment system using VDM++. Lecture Notes in Computer Science, pp. 163-178, 2012.

[35] Lindfeldt, O., The impact of platform screen doors on rail capacity. International Journal of Transport Development and Integration, 1(3), pp. 601-10, 2017.

[36] Lecomte, T., Servat, T. \& Pouzancre, G., Formal methods in safety-critical railway systems. Symposium on Formal Methods, 2010.

[37] Heinrich, M. et al., Security requirements engineering in safety-critical railway signalling networks. Security and Communication Networks, pp. 1-14, 2019.

[38] Venkatesh, V., Morris, M., Davis, G. \& Davis, F., User acceptance of information technology: Toward a unified view. MIS Quarterly, 27(3), pp. 425-78, 2003. 